\title{
Membrane trafficking inspired hydrolysis of non-activated esters at physiological pH
}

Raki Mandal†, Kingshuk Mahanty†े, Subhendu Mandal, Suman De Sarkar*, and Pradip K. Tarafdar*

Department of Chemical Sciences, Indian Institute of Science Education and Research Kolkata, Mohanpur-741246, India

$\dagger$ Both authors contributed equally

*Corresponding author:

Dr. Pradip Kumar Tarafdar

Department of Chemical Sciences

Indian Institute of Science Education and Research Kolkata

Mohanpur-741 246, India

E-mail: tarafdar@iiserkol.ac.in

Dr. Suman De Sarkar

Department of Chemical Sciences

Indian Institute of Science Education and Research Kolkata

Mohanpur-741 246, India

E-mail: sds@iiserkol.ac.in 


\begin{abstract}
Natural enzymes establish the proximity of the substrates to perform challenging reactions in aqueous medium, whereas most chemical catalysts typically follow stochastic way and are less efficient. Inspired by the membrane trafficking, a core biological process, herein we report that positively charged micro heterogeneous vehicles loaded with substrate could be trafficked suitably at the site of the reaction to promote the localization and proximity of the reactants. The guided vehicular delivery coupled with electrolysis overcomes the entropic barrier related to the proximity of the reactants and allows the hydrolysis of non-activated esters at physiological $\mathrm{pH}$. Mechanistic investigations suggest that the reaction utilizes the electrochemical energy to generate hydroxide ion at the cathode and the positively charged micellar vehicles (loaded with substrates) trafficked selectively near cathode to promote the hydrolysis. The in situ modulation of surface charge was exploited to accelerate or inhibit the hydrolysis in a controlled manner akin to cofactors or zymogens of natural enzymes. We believe this elegant membrane trafficking inspired approach paves the way for the further applications of proximity controlled selective transformations in organic synthesis using green aqueous medium.
\end{abstract}

\title{
Main
}

Nature has evolved efficient chemical processes and uses enzymes to promote otherwise impossible reactions ${ }^{1}$. The precise control of natural enzymes is necessary for the regulation of various biological processes such as metabolism, signal transduction, and cell growth. These enzymes are recognized as one of the most effective catalysts due to their high affinity towards substrates, superior catalytic efficiency, and unrivaled selectivity ${ }^{2}$. Due to their tremendous importance in chemical and biotechnological processes ${ }^{3}$, efforts have been made to create synthetic 
enzymes $^{4}$, small molecule bioinspired robust catalysts ${ }^{5}$. However, designing an synthetic enzyme is challenging, synthesis is laborious and the activity is often limited in industrial use ${ }^{6}$. Therefore, new designs of cheap and novel biomimetics are necessary ${ }^{7}$. Over the last few decades, scientists have extensively investigated to understand the superiority of natural enzymes and uncovered various important aspects of enzymatic reactions. Although factors like the role of the electric field ${ }^{8,9}$, promiscuity of the enzyme active site $^{10}$ are still emerging, there is wide consensus that the close proximity of reactants, high local concentration, the release of products are the several important strategies for an efficient enzymatic activity ${ }^{11}$. Close proximity and high local concentration of the reactants are closely related and responsible to overcome the entropic barrier involved in the reaction. Unfortunately, a small molecule enzyme-mimic finds difficulty to maintain proximity and localization of the reactants due to the entropic penalties.

Mother nature is always the inspiration to the scientists behind many important discoveries. To deliver the proteins, metabolites, neurotransmitters and other biological substrates at the active site and protect those from unwanted chemical reactions, nature evolved membrane trafficking in higher order organisms. Membrane trafficking is a core biological process and immensely important in neurotransmitter release, viral infection, and other cellular processes ${ }^{12-18}$. The intracellular trafficking pathways like ER to Golgi or Golgi to ER are generally specific and encoded by SNARE proteins involved in particular trafficking ${ }^{19-21}$. With the help of particular membrane trafficking pathway nature directs the correct cargo in a guided manner i.e. to the right place at the right time. Therefore, we envisioned whether the membrane trafficking can be utilized to promote proximity and localization of reactants to design a small molecule enzyme mimic.

Amongst the pool of enzymes, esterase is an important academic and industrial enzyme plays enormous role in vivo and also in food, paper manufacturing, detergent formulations and 
pharmaceuticals industry ${ }^{22,23}$. While an ester usually be hydrolyzed at basic $\mathrm{pH}$ and at elevated temperature, the catalytic triad of esterase performs similar reaction at neutral buffer solution $(\mathrm{pH}$ 7.0) and at physiological temperature ${ }^{24,25}$. Researchers put considerable effort to design the synthetic mimic of esterase using de novo design ${ }^{26,27}$, dendrimer ${ }^{28}$, polymer $^{29}$, membrane ${ }^{30,31}$, selfassembling peptides ${ }^{32,33}$, nanofibers ${ }^{34}$ and amyloid fibrils ${ }^{5,33,35}$ to cleave an ester at the physiological condition. However, most of the designs are only able to cleave the active ester like $p$-nitrophenol ester ${ }^{5,27,29-36}$ and remain ineffective towards normal non-activated esters ${ }^{37}$. Thus, it is still challenging to hydrolyze an unactivated ester at physiological $\mathrm{pH}$ and new methods are being developed ${ }^{37}$. To circumvent this deficiency and to overcome the entropic barrier related to the localization and proximity of the reactants, we opt to use membrane trafficking to design a biomimetic esterase. Although supramolecular interactions were widely used to achieve primary and secondary interaction of designed biomimetic esterase, the guided vehicular trafficking of the substrate to the active site was not utilized. To hydrolyze an ester at physiological $\mathrm{pH}$, we hypothesized to traffic the substrate selectively towards a region having a high local concentration of hydroxide ion while maintaining the neutral $\mathrm{pH}$ of the bulk solution. We planned to utilize the electrochemical energy to generate high local hydroxide ion concentration at the cathode and selectively direct the substrates towards the cathode using positively charged micellar vehicles. We envisioned that the guided vehicular delivery of the substrate near cathode will establish localization and proximity of esters and hydroxide, thus will promote the hydrolysis of the nonactivated esters at overall physiological pH (Fig. 1A). On the other hand, the negatively charged and neutral micro heterogeneous vehicles may be labeled as the misguided one, they should not selectively traffic the substrate towards the cathode, and should be ineffective to hydrolyze the ester under similar condition (Fig. 1A). We also hypothesized that in situ surface charge 
modulation of the vehicles may convert the system from a guided to misguided one or vice versa and therefore can be utilized to accelerate or inhibit the hydrolysis in a controlled manner akin to the cofactors or zymogens of natural enzymes. Based on these concepts, in the present study, we have utilized several important advantages of the aqueous green environment over the other organic solvents. The aqueous environment helps to build up the vehicles via self-assembly ${ }^{38,39}$, loads of the substrate into vehicles ${ }^{40}$, guides the vehicles to the delivery site, supplies the hydroxide ion by the electrochemical reduction of water ${ }^{41,42}$. These advantages stimulate the hydrolysis at mild physiological condition, which could not be achieved under similar condition in other solvents. We believe that our simple membrane trafficking inspired approach paves the way for the further application to proximity related electron transfer reactions to selectively generate radical or ionic intermediates for organic synthesis in the green aqueous medium.
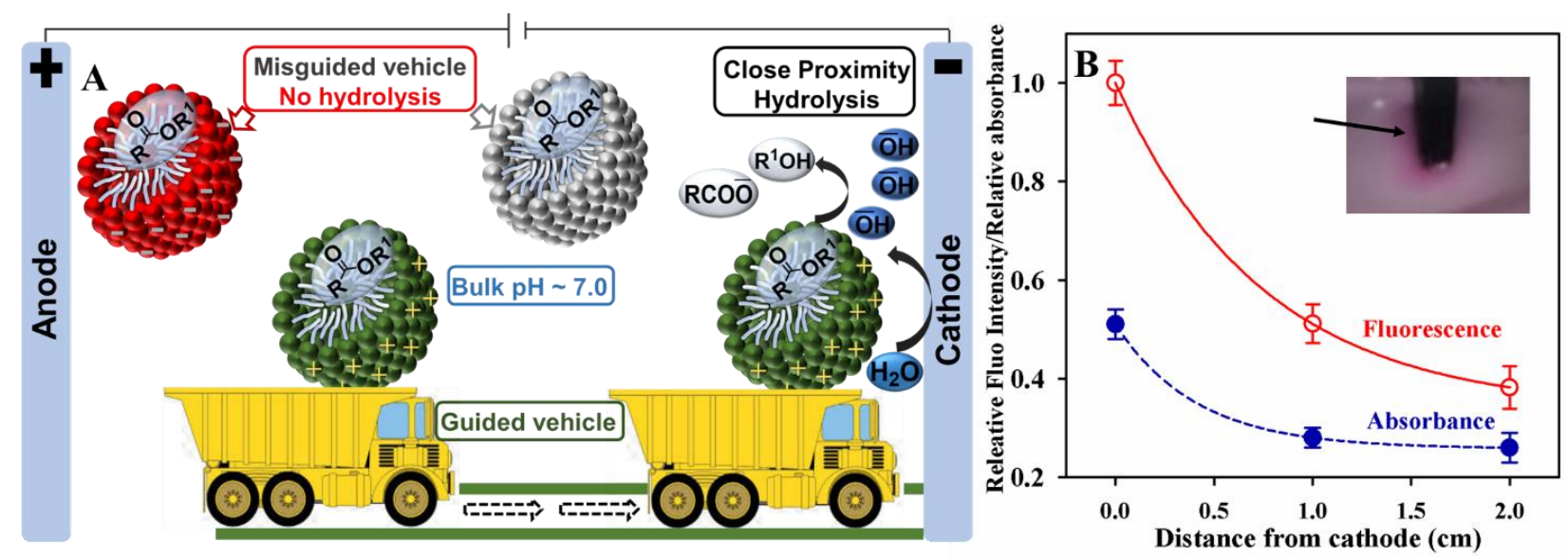

Fig. 1: A) Schematic model on the guided vehicular trafficking of the substrate to promote proximity of reactants and subsequent hydrolysis. The positively charged (green) vehicles trafficked to cathode, the negatively charged (red) vehicles trafficked to anode and neutral (grey) vehicles follows diffusion-controlled movement. B) Rhodamine PE doped (0.1 mol\%) CTAB micelles trafficked selectively near the cathode. 


\section{Results and Discussion}

To verify the aforementioned hypothesis, rhodamine-conjugated phosphatidylethanolamine (RhPE) was incorporated into the positively charged cetyltrimethylammonium bromide (CTAB) micelles $(0.1 \mathrm{~mol} \%)$ and the micelles were suspended into $\mathrm{pH} 7.0$ tris buffer. A constant current was applied using graphite electrodes to check whether the positively charged micelles trafficked towards the cathode. The fluorescence intensity and absorbance of Rh-PE doped CTAB micellar solutions at various distance from the cathode were measured and the observations support that CTAB-doped micelles are localized near to the cathode (Fig. 1B). Visual inspection of the solution also supports the localization of Rh-PE doped CTAB micelles near cathode (inset, Fig. 1B). The Rh-PE also incorporated into neutral triton $\mathrm{X}-100$ micelles $(0.1 \mathrm{ml} \%)$ and no localization of micelles near cathode was observed. This suggests that electrostatic factors guide the micelle trafficking towards the cathode and using the approach the delivery of a substrate (e.g. ester) near the cathode is possible where the CTAB/positively charged micelles act as guided vehicles. The advantage of the guided vehicular delivery is of two-fold. First, it facilitates the proper transport of the substrate at the desired place and second, using the micellar vehicle, a high local concentration of the reactants can also be achieved. Using the guided vehicular delivery inspired by membrane trafficking, ester hydrolysis at a neutral buffer was planned. To perform an ester hydrolysis availability of hydroxide ion near the cathode surface is required. Therefore, electrochemical reduction of water at $\mathrm{pH} 7.0$ was carried out to generate $\mathrm{OH}^{-}$ions and their localization near cathode was monitored by adding a pinch of phenolphthalein solution. The pink color appeared near to cathode which suggests that the local $\mathrm{OH}^{-}$concentration was much higher close to the cathode than other locations of the electrolytic cell (Fig. S1). Therefore, the membrane trafficking guided transport of substrate and the electrolytic method likely help to localize the 
reactants at close proximity. The close proximity is a wonderful strategy not only used by natural enzymes but also applied to many organic reactions ${ }^{43}$.
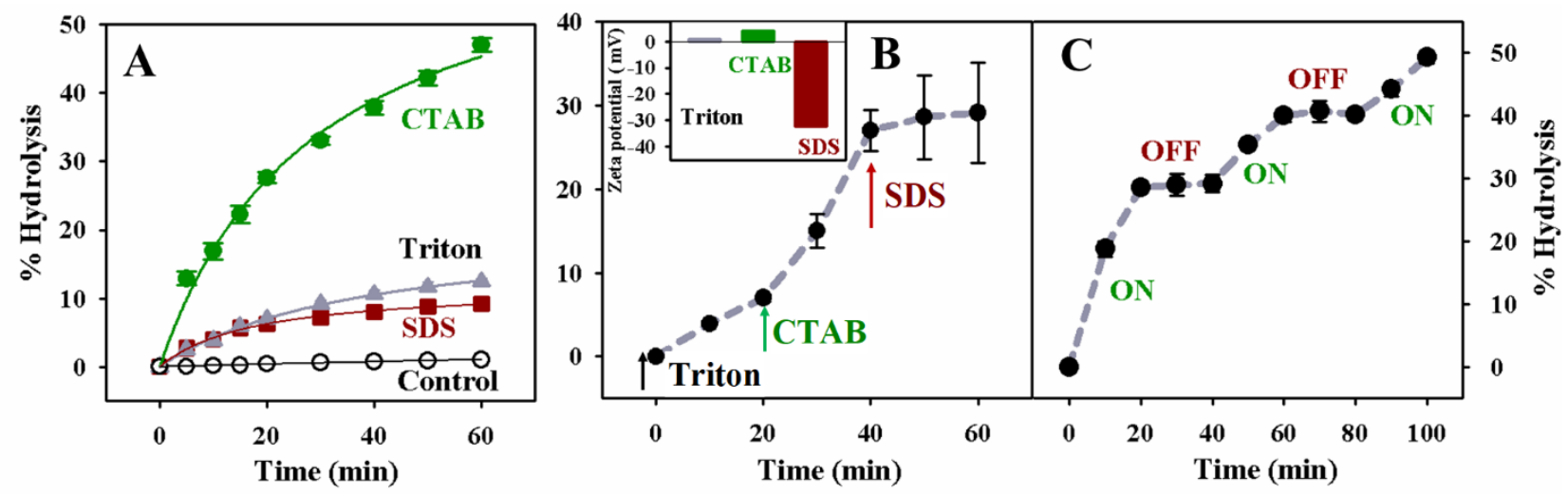

Fig.2: Kinetics of the hydrolysis of butyl tribromophenol ester. A) Hydrolysis of ester in different types of vehicles: positively charge CTAB, negatively charged SDS and neutral Triton X-100. B) Hydrolysis may be initiated or inhibited in situ by the addition of different types of surfactant; inset: zeta potential change by the addition of surfactants, C) Role of constant current on the extent of hydrolysis.

To take advantage of close proximity and high local concentration of the reactants, we tested the hydrolysis of a tribromophenol ester, a relatively less activated model ester (as compared to p-nitro phenolic ester). Butyl tribromophenol ester was synthesized and incorporated into $3.6 \mathrm{wt} \% \mathrm{CTAB}$ at $\mathrm{pH} 7.0$ tris buffer. Upon applying $10 \mathrm{~mA}$ current into the solution, we measured the absorbance at $320 \mathrm{~nm}$ in a time-dependent manner. The increase in absorbance suggests that the tribromophenol ester was hydrolyzed in the presence of CTAB micelles (Fig. 2A). Now to check the role of trafficking inspired guided delivery using CTAB micelles, the same ester was placed in the electrolytic chamber at $\mathrm{pH} 7.0$ (no micelles) and current was applied. It was found that in the absence of CTAB the ester remains stable (Fig. 2A) supporting the role of CTAB mediated trafficking of micelles near the cathode. To verify the role of micellar catalysis, the ester was 
incorporated into sodium dodecyl sulfate (SDS) and triton X-100 (neutral) micelles at pH 7.0 buffer and the current was applied. The results suggest that the tribromophenol ester remains mostly stable $(<10 \%$ conversion) when incorporated into a misguided vehicle like anionic SDS and neutral triton X-100 micelles and therefore, the ester hydrolysis can be tuned by choosing suitable trafficking inspired guided delivery (Fig. 2A). Apart from the superior catalytic activity of natural enzymes another very important aspect is the regulation of catalytic activity, which largely controlled by small binding ligands, temperature, $\mathrm{pH}$, light, cofactors and zymogens. For example, many blood coagulation factors possess little hydrolytic activity which greatly enhanced upon binding to a negatively charged membrane ${ }^{44}$. Therefore, tunability of the catalytic efficiency of a catalyst is very much important and useful in on-demand enhanced activity. Since our trafficking inspired guided delivery may be tuned by controlling the efficiency of guided delivery over the misguided one (Fig. 2A), it may be used as a molecular brake or accelerator to control the hydrolysis. The hydrolysis of the ester was started with neutral triton X-100 vehicle and as mentioned earlier that the kinetics was slow (Fig. 2A, 2B). Interestingly, the addition of CTAB into the solution (in situ) greatly enhances the rate of hydrolysis (Fig. 2B) clearly demonstrating the role of guided delivery of substrate and the role of the positively charged surface. The zeta potential of the triton X-100 micelle and after addition of CTAB was measured which suggests that the zeta potential becomes more positive upon the addition of CTAB (Fig. 2B, inset). Next, SDS was added into the solution to tune the surface into negatively charged (negative zeta potential, inset Fig. 2B), which almost block the hydrolysis. Therefore, by tuning the composition of the micelle transform a misguided vehicle to guided one or vice versa and the esterase activity may be controlled. To the best of our knowledge, the examples of real-time in situ molecular brake and accelerator to control a reaction is limited. We checked further whether the esterase activity 
can be regulated by the current? As expected, it was found that when the electricity is ON it exhibits esterase activity and after switching OFF the current, the esterase mimic stops working (Fig. 2C). This experiment also suggests that the uninterrupted flow of electricity is required and one-time generation of hydroxide ions is not sufficient to run the hydrolysis continuously. It appears that the generated hydroxide may end up to anode for oxidation and continuous current flow is required to maintain the production in cathode. Cetyltrimethylammonium bromide (CTAB) was chosen as the guided vehicle over cetyltrimethylammonium chloride (CTAC) as bromide can be oxidized over chloride ions at lower potential. Therefore, bromide may compete better with hydroxide for oxidation in anode and hydroxide localization likely be enhanced nearer to the cathode.

The guided vehicular delivery inspired by membrane trafficking coupled with electrochemical reaction efficiently breaks the relatively active esters (tribromophenol). Next, we tested the applicability of the method for the hydrolysis of more challenging unactivated aliphatic esters. Benzyl ester of butyric acid (1ab) as a stable aliphatic ester was incorporated into CTAB micelles and placed in an undivided cell comprised with graphite electrode in tris buffer (pH 7.0) at $25^{\circ} \mathrm{C}$. A constant current of $20 \mathrm{~mA}$ in was applied and the hydrolysis product benzyl alcohol was obtained in 4 hours with $58 \%{ }^{1} \mathrm{H}$ NMR yield (table 1, entry 1). Lowering the current density to $10 \mathrm{~mA}$ and increasing to $25 \mathrm{~mA}$ led to the reduction in yield (entries 2-3) and $20 \mathrm{~mA}$ current was applied for further optimization. Slightly diminished yield was observed when water was used as the solvent with adding the tris buffer (entry 4). Various other electrolytes such as lithium perchlorate, tetrabutyl ammonium tetrafluroborate were screened in water solvent and proved to be inefficient (entries 5-6). Replacing water with acetonitrile solvent with lithium perchlorate as supporting electrolytes significantly suppressed the hydrolysis (entry 7) suggesting the role of guided vehicle 
Table 1: Optimization of the guided ester hydrolysis conditions ${ }^{a}$

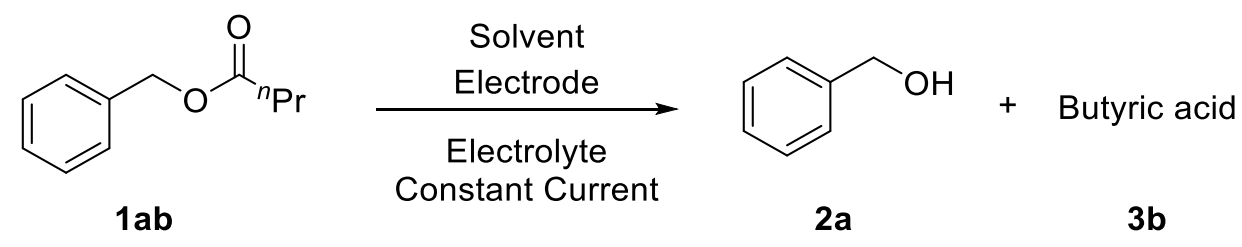

\begin{tabular}{|c|c|c|c|c|c|}
\hline Entry & Solvent & Electrode & Electrolyte & Current & Yield $^{b}$ \\
\hline 1 & pH 7 buffer & $\mathrm{C}(+) / \mathrm{C}(-)$ & CTAB & $20 \mathrm{~mA}$ & $58 \%$ \\
\hline 2 & pH 7 buffer & $\mathrm{C}(+) / \mathrm{C}(-)$ & CTAB & $10 \mathrm{~mA}$ & $52 \%$ \\
\hline 3 & pH 7 buffer & $\mathrm{C}(+) / \mathrm{C}(-)$ & CTAB & $25 \mathrm{~mA}$ & $51 \%$ \\
\hline 4 & $\mathrm{H}_{2} \mathrm{O}$ & $\mathrm{C}(+) / \mathrm{C}(-)$ & CTAB & $10 \mathrm{~mA}$ & $40 \%$ \\
\hline 5 & $\mathrm{H}_{2} \mathrm{O}$ & $\mathrm{C}(+) / \mathrm{C}(-)$ & $\mathrm{LiClO}_{4}$ & $10 \mathrm{~mA}$ & ND \\
\hline 6 & $\mathrm{H}_{2} \mathrm{O}$ & $\mathrm{C}(+) / \mathrm{C}(-)$ & $\mathrm{Bu}_{4} \mathrm{NBr}$ & $10 \mathrm{~mA}$ & ND \\
\hline 7 & $\mathrm{MeCN}$ & $\mathrm{C}(+) / \mathrm{C}(-)$ & $\mathrm{LiClO}_{4}$ & $10 \mathrm{~mA}$ & ND \\
\hline 8 & pH 7 buffer & $\mathrm{C}(+) / \mathrm{Ni}(-)$ & CTAB & $20 \mathrm{~mA}$ & $35 \%$ \\
\hline 9 & pH 7 buffer & $\mathrm{C}(+) / \mathrm{RVC}(-)$ & CTAB & $20 \mathrm{~mA}$ & $52 \%$ \\
\hline 10 & pH 7 buffer & $\mathrm{C}(+) / \mathrm{Pt}(-)$ & CTAB & $20 \mathrm{~mA}$ & $20 \%$ \\
\hline $11^{c}$ & pH 7 buffer & $\mathrm{C}(+) / \mathrm{C}(-)$ & CTAB & $20 \mathrm{~mA}$ & $79 \%(77 \%)^{d}$ \\
\hline
\end{tabular}

${ }^{a}$ Reaction conditions: 1ab $(0.2 \mathrm{mmol})$, electrolyte $((3.6 \mathrm{wt} \%$ or $0.1 \mathrm{M})$ in solvent $(8 \mathrm{~mL})$, in an undivided cell with constant current $=10-25 \mathrm{~mA}$, at r.t. under air for 4 h. ${ }^{b 1} \mathrm{H}$ NMR yield. ${ }^{a}$ Reaction was conducted without stirring. ${ }^{d}$ Yield of pure product. 
delivery coupled with electrolytic reaction. Replacing the graphite electrode with nickel foam, RVC and platinum were found less effective (entries 8-10). Since the reaction was driven by guided vehicular delivery to establish close proximity and localization of reactants, we hypothesized that the stirring of the solution (general practice in any solution-phase chemical reaction) likely perturbs the effective trafficking. Indeed, when the constant mixing of the reaction mixture was stopped, the extent of the hydrolysis reaction was amplified considerably to $77 \%$ of the isolated yield (entry 11). To ensure the practicality of the guided delivery coupled with electrolysis, the reaction was performed in 1 gram scale. The reaction proceeded smoothly to achieve $65 \%$ of the isolated yield.

The reaction scope was extended to other substrates affording hydrolysis products in good to excellent yields (Fig. 3). Esters having different combinations of aliphatic acids and benzylalcohol derivatives as well as 1-napthalenemethanol were found to be suitable for the guided hydrolysis. However, with the increase in the acyl chain length of the fatty acid ( $n=2$ to 8$)$ the yield slightly decreased. Next, lithocholic acid methyl ester (1e), ibuprofen methyl ester (1f), amino acid ester (1g), and trihexanoyl glycerol (1h) were also hydrolyzed as model bioactive esters in good yields (Fig. 3). These results demonstrate the general applicability of our simple guided vehicular delivery approach to hydrolyze various unactivated esters at room temperature in physiological buffer. After establishing the optimized condition and broad applicability of substrates, the recyclability of the method was tested. Organic layer of the reaction mixture was extracted out and the aqueous layer along with the surfactant was reused for the next cycle. Thereafter substrate (1ab) was added to the aqueous medium and electrolyzed further using standard electrochemical condition but surprisingly, it did not afford any desired product. As switching off the current stop hydrolysis (Fig. 2C), it was mentioned above about the necessity of continuous generation of 
hydroxide ion and the possible role of bromide oxidation to maintain the localization and high concentration of hydroxide ion near the cathode. We hypothesized that the bromide ions of CTAB get oxidized after the first cycle, as a result no product after the first cycle was obtained. Therefore, the addition of a bromide salt $(\mathrm{NaBr})$ to the reaction mixture was expected to recharge the cationic surfactant and gratifyingly $65 \%$ of the product was obtained in the second cycle. The same procedure was repeated and in the third cycle 51\% product was obtained (Fig. S2). It was found that under the experimental condition bromide ion was oxidized to non-oxidizable bromate ion $\left(\mathrm{BrO}_{3}\right)$ (detected by mass spectrometry and qualitative analysis, see SI, Fig. S3) $)^{45}$, which is incapable to compete with hydroxide ion for oxidation. Thus, recharging with $\mathrm{NaBr}$ is required before each cycle. The recyclability of the guided vehicular delivery coupled to electrolytic reaction greatly enhances the applicability to hydrolyze esters at the physiological condition. 


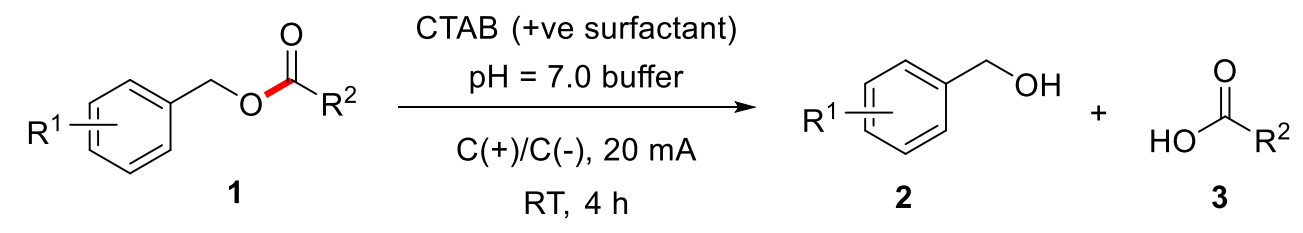

Benzyl ester of short chain acids

Benzyl ester of long chain acids<smiles>CC(=O)OCc1ccccc1</smiles>

1aa, $81 \%$<smiles>CCCC(=O)OCc1ccccc1</smiles>

1ab, $77 \%$<smiles>CC(C)C(=O)OCc1ccccc1</smiles>

1 ac, $69 \%$<smiles>CCCC(=O)OCc1ccccc1</smiles>

1ad, $70 \%$

Gram Scale: $65 \%$<smiles>CC(=O)OCc1ccc(F)cc1</smiles>

1ba, $92 \%$<smiles>CC(=O)OCc1cccc2ccccc12</smiles>

1ca, $82 \%$<smiles>CCCC(=O)OCc1ccc(F)cc1</smiles>

1bb, $78 \%$<smiles>CC(C)C(=O)OCc1cccc2ccccc12</smiles>

1cb, $73 \%$<smiles>CC(C)C(=O)OCc1ccc(F)cc1</smiles>

1bc, $71 \%$<smiles>CC(C)C(=O)OCc1cccc2ccccc12</smiles>

$1 \mathrm{cc}, 65 \%$<smiles>CCCC(=O)OCc1ccc(F)cc1</smiles>

1bd, $60 \%$<smiles>CCC(=O)OCc1cccc2ccccc12</smiles>

$1 \mathrm{~cd}, 61 \%$<smiles>COC(=O)CC[C@@H](C)[C@H]1CC[C@H]2[C@@H]3CCC4C[C@@H](O)CC[C@]4(C)[C@H]3CC[C@]21C</smiles>

1 e, $67 \%$

Methyl lithocolate

Cholesterol Ester

Esters of biologically relevant molecules<smiles>COC(=O)C(C)c1ccc(CC(C)C)cc1</smiles>

1f, $64 \%$

Methyl ester of ibuprofen

Pro-drug Ester<smiles>O=C(CC(NC(=O)OCc1ccccc1)C(=O)O)OCc1ccccc1</smiles>

$1 \mathrm{~g}, 58 \%$

Aspartic Benzyl Ester

Amino acid Ester

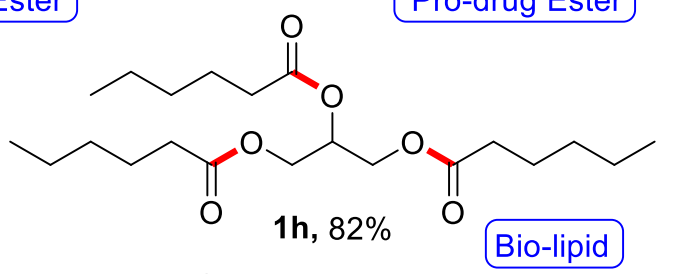

Glycerol trihexanoate

Fig. 3: The synthetic scope of the guided vehicular delivery inspired hydrolysis of esters. 


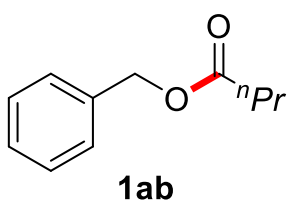

A)

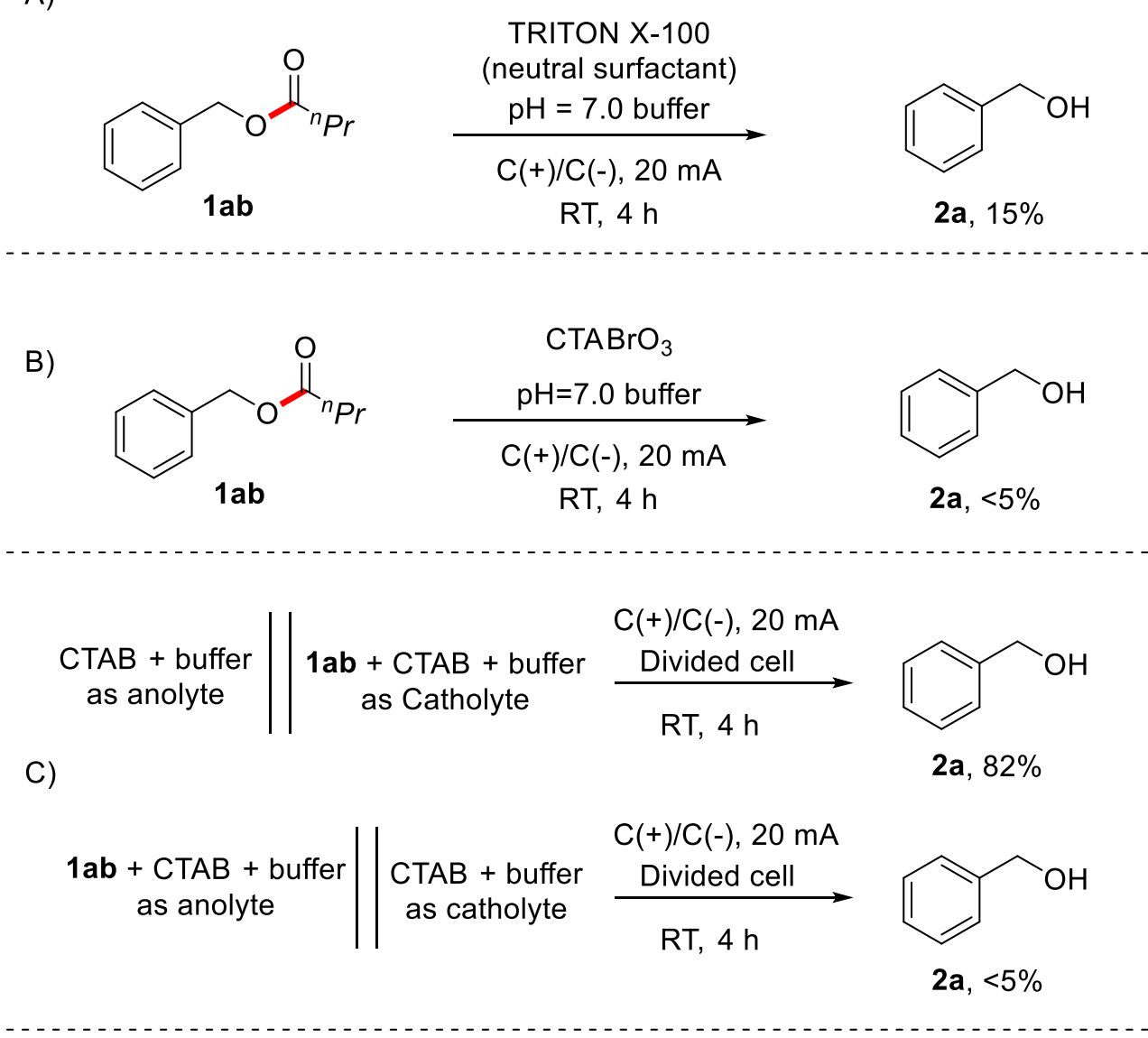

$$
\begin{gathered}
\begin{array}{c}
\text { SDS (-ve surfactant) } \\
\mathrm{pH}=7.0 \text { buffer }
\end{array} \\
\underset{\mathrm{C}(+) / \mathrm{C}(-), 20 \mathrm{~mA}}{\longrightarrow}
\end{gathered}
$$$$
\mathrm{RT}, 4 \mathrm{~h}
$$<smiles>OCc1ccccc1</smiles>

$2 a,<5 \%$

Fig. 4: Mechanistic investigations of guided trafficking inspired hydrolysis. The role of various A) surfactants and B) counterion on the extent of hydrolysis of 1ab. C) Control experiments at divided cell suggest that the localization of $\mathrm{CTAB}$ near cathode is necessary for successful hydrolysis.

To understand the detailed mechanistic overview of the protocol, an array of control experiments was performed (Table 1). Similar to the tribromophenol ester the hydrolysis of benzyl butyrate ester (1ab) was hampered by switching off the output power supply and while loaded into a 
misguided vehicle such as SDS and Triton X-100 (Fig. 1A, Fig. 4). It was again evidenced that the contentious generation of the hydroxide ion and thus maintaining a persistence basic environment near to the cathode is the key factor to perform the hydrolysis. Thus, the use of non-oxidizable counter anion like $\mathrm{BrO}_{3}^{-}$breaks this system and ineffective hydrolysis was observed (Fig. 4).

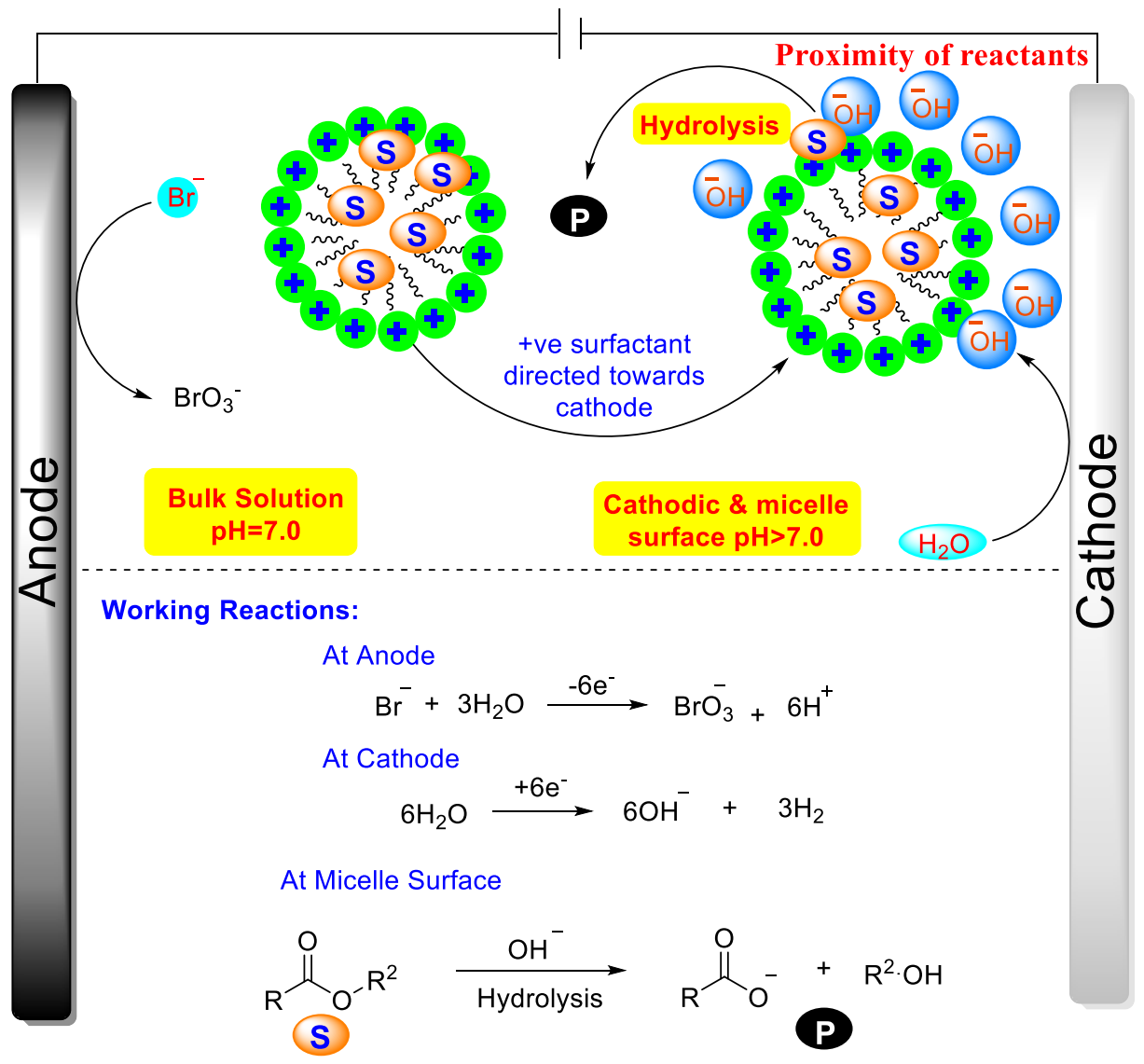

Fig. 5: Proposed mechanism of the guided vehicular delivery of ester and hydrolysis near the cathode.

The hydrolysis of ester $\mathbf{1 a b}$ was further carried out in divided cell by altering the location of the substrate (Fig. 4). As expected, the ester hydrolysis was observed only when the substrate 1ab was taken in the cathodic chamber. This clearly suggests that the hydrolysis is occurring only close to 
the cathode and also demonstrates the importance of guided vehicular delivery of substrate at a particular site to carry out the desired reaction. The targeted transformation was not successful without the vehicle (no micelles) and with misguided vehicles (negatively charged and neutral micelles). On the basis of the mechanistic as well as the optimization studies, a mechanism of the guided ester hydrolysis is proposed (Fig. 5). All the experiments confirm the decisive role of directed vehicular delivery of the substrate inside the micro-structured environment to perform the hydrolysis (Fig. 5). Thus, physiological aqueous medium becomes superior than any other solvents. It helps to build the vehicles via self-assembly, to load of the substrate into the vehicles, guide the vehicles to promote proximity and supply the hydroxide by the electrochemical reduction of water.

\section{Conclusion}

In summary, the membrane trafficking inspired guided vehicular delivery was coupled with electrolysis to perform hydrolysis of non-activated esters at physiological buffer. The vehicular delivery can be tuned externally by additives and thus were able to accelerate and inhibit the hydrolysis in situ. Interestingly, the aqueous green environment was superior to other organic solvents as it facilitates the vehicular trafficking and supplies the hydroxide ions in close proximity of the substrates to promote the hydrolysis. The elegant approach may further be extended to other acid or base promoted reactions as well as to electron transfer reactions via the guided delivery of the substrate close to the electrodes in aqueous medium.

\section{Acknowledgements}


PKT acknowledges support from DST-Inspire (IFA13-CH-120, 2014) and the Science and Engineering Research Board (SERB) Early Career Award (ECR/2016/001935). RM thanks CSIR and KM thanks UGC for fellowship. PKT and SDS thanks IISER Kolkata for infrastructure and financial support.

\section{References}

1. Arnold, F. H. Directed Evolution: Bringing New Chemistry to Life. Angew. Chemie - Int. Ed. 57, 4143-4148 (2018).

2. Benkovic, S. J. \& Hammes-Schiffer, S. A perspective on enzyme catalysis. Science 301, 1196-1202 (2003).

3. Schmid, A. et al. Industrial biocatalysis today and tomorrow. Nature 409, 258-268 (2001).

4. Dong, Z., Luo, Q. \& Liu, J. Artificial enzymes based on supramolecular scaffolds. Chemical Society Reviews 41, 7890-7908 (2012).

5. Makam, P. et al. Non-proteinaceous hydrolase comprised of a phenylalanine metallosupramolecular amyloid-like structure. Nature Catalysis 2, 977-985 (2019).

6. Arnold, F. H. Combinatorial and computational challenges for biocatalyst design. Nature 409, 253-257 (2001).

7. Chen, K. \& Arnold, F. H. Engineering new catalytic activities in enzymes. Nature Catalysis 1-11 (2020). doi:10.1038/s41929-019-0385-5 
8. Fried, S. D. \& Boxer, S. G. Electric Fields and Enzyme Catalysis. Annu. Rev. Biochem. 86, 387-415 (2017).

9. Fried, S. D., Bagchi, S. \& Boxer, S. G. Extreme electric fields power catalysis in the active site of ketosteroid isomerase. Science 346, 1510-1514 (2014).

10. Leveson-Gower, R. B., Mayer, C. \& Roelfes, G. The importance of catalytic promiscuity for enzyme design and evolution. Nature Reviews Chemistry 3, 687-705 (2019).

11. Voet, D., Voet, J. G. \& Pratt, C. W. Fundamentals of biochemistry: life at the molecular level.

12. Harrison, S. C. Viral membrane fusion. Nature Structural and Molecular Biology 15, 690-698 (2008).

13. Yang, S. T. et al. HIV virions sense plasma membrane heterogeneity for cell entry. Sci. Adv. 3, e1700338 (2017).

14. Tarafdar, P. K., Chakraborty, H., Bruno, M. J. \& Lentz, B. R. PhosphatidylserineDependent Catalysis of Stalk and Pore Formation by Synaptobrevin JMR-TMD Peptide. Biophys. J. 109, 1863-1872 (2015).

15. Takamori, S. et al. Molecular Anatomy of a Trafficking Organelle. Cell 127, 831-846 (2006).

16. Südhof, T. C. The molecular machinery of neurotransmitter release (nobel lecture). Angewandte Chemie - International Edition 53, 12696-12717 (2014).

17. Zick, M. \& Wickner, W. T. A distinct tethering step is vital for vacuole membrane fusion. 
Elife 3, e03251 (2014).

18. Finger, F. P. \& White, J. G. Fusion and fission: Membrane trafficking in animal cytokinesis. Cell 108, 727-730 (2002).

19. Baker, R. W. \& Hughson, F. M. Chaperoning SNARE assembly and disassembly. Nature Reviews Molecular Cell Biology 17, 465-479 (2016).

20. Jahn, R. \& Scheller, R. H. SNAREs - Engines for membrane fusion. Nature Reviews Molecular Cell Biology 7, 631-643 (2006).

21. McNew, J. A. et al. Compartmental specificity of cellular membrane fusion encoded in SNARE proteins. Nature 407, 153-159 (2000).

22. Hasan, F., Shah, A. A. \& Hameed, A. Industrial applications of microbial lipases. Enzyme Microb. Technol. 39, 235-251 (2006).

23. Panda, T. \& Gowrishankar, B. S. Production and applications of esterases. Appl. Microbiol. Biotechnol. 67, 160-169 (2005).

24. Rauwerdink, A. \& Kazlauskas, R. J. How the Same Core Catalytic Machinery Catalyzes 17 Different Reactions: The Serine-Histidine-Aspartate Catalytic Triad of $\alpha / \beta$-Hydrolase Fold Enzymes. ACS Catalysis 5, 6153-6176 (2015).

25. Hedstrom, L. Serine protease mechanism and specificity. Chem. Rev. 102, 4501-4523 (2002).

26. Huang, P. S., Boyken, S. E. \& Baker, D. The coming of age of de novo protein design. Nature 537, 320-327 (2016). 
27. Song, W. J. \& Tezcan, F. A. A designed supramolecular protein assembly with in vivo enzymatic activity. Science 346, 1525-1528 (2014).

28. Kofoed, J. \& Reymond, J. L. Dendrimers as artificial enzymes. Current Opinion in Chemical Biology 9, 656-664 (2005).

29. Nothling, M. D. et al. Simple Design of an Enzyme-Inspired Supported Catalyst Based on a Catalytic Triad. Chem 2, 732-745 (2017).

30. Chadha, G. \& Zhao, Y. Histidine-functionalized water-soluble nanoparticles for biomimetic nucleophilic/general-base catalysis under acidic conditions. Org. Biomol. Chem. 11, 6849-6855 (2013).

31. Poznik, M. \& König, B. Cooperative hydrolysis of aryl esters on functionalized membrane surfaces and in micellar solutions. Org. Biomol. Chem. 12, 3175-3180 (2014).

32. Zhang, C. et al. Switchable Hydrolase Based on Reversible Formation of Supramolecular Catalytic Site Using a Self-Assembling Peptide. Angew. Chemie - Int. Ed. 56, 1451114515 (2017).

33. Rufo, C. M. et al. Short peptides self-assemble to produce catalytic amyloids. Nat. Chem. 6, 303-309 (2014).

34. Guler, M. O. \& Stupp, S. I. A self-assembled nanofiber catalyst for ester hydrolysis. J. Am. Chem. Soc. 129, 12082-12083 (2007).

35. Sarkhel, B., Chatterjee, A. \& Das, D. Covalent Catalysis by Cross $\beta$ Amyloid Nanotubes. J. Am. Chem. Soc. jacs.9b13517 (2020). doi:10.1021/jacs.9b13517 
36. Singh, N., Conte, M. P., Ulijn, R. V., Miravet, J. F. \& Escuder, B. Insight into the esterase like activity demonstrated by an imidazole appended self-assembling hydrogelator. Chem. Commun. 51, 13213-13216 (2015).

37. Rodon Fores, J. et al. Supported Catalytically Active Supramolecular Hydrogels for Continuous Flow Chemistry. Angew. Chemie - Int. Ed. 58, 18817-18822 (2019).

38. Lipshutz, B. H., Ghorai, S. \& Cortes-Clerget, M. The Hydrophobic Effect Applied to Organic Synthesis: Recent Synthetic Chemistry “in Water”. Chemistry - A European Journal 24, 6672-6695 (2018).

39. Cortes-Clerget, M., Lee, N. R. \& Lipshutz, B. H. Synthetic chemistry in water: applications to peptide synthesis and nitro-group reductions. Nat. Protoc. 14, 1108-1129 (2019).

40. Giedyk, M. et al. Photocatalytic activation of alkyl chlorides by assembly-promoted single electron transfer in microheterogeneous solutions. Nature Catalysis 3, 40-47 (2020).

41. Chen, L., Dong, X., Wang, Y. \& Xia, Y. Separating hydrogen and oxygen evolution in alkaline water electrolysis using nickel hydroxide. Nat. Commun. 7, 1-8 (2016).

42. Singh, M. R., Kwon, Y., Lum, Y., Ager, J. W. \& Bell, A. T. Hydrolysis of Electrolyte Cations Enhances the Electrochemical Reduction of $\mathrm{CO} 2$ over $\mathrm{Ag}$ and $\mathrm{Cu}$. J. Am. Chem. Soc. 138, 13006-13012 (2016).

43. Das, P., Kumar, A., Howlader, P. \& Mukherjee, P. S. A Self-Assembled Trigonal Prismatic Molecular Vessel for Catalytic Dehydration Reactions in Water. Chem. - A Eur. J. 23, 12565-12574 (2017). 
44. Qureshi, S. H., Yang, L., Manithody, C. \& Rezaie, A. R. Membrane-dependent interaction of factor $\mathrm{Xa}$ and prothrombin with factor $\mathrm{Va}$ in the prothrombinase complex. Biochemistry 48, 5034-5041 (2009).

45. Cettou, P., Robertson, P. M. \& Ibl, N. On the electrolysis of aqueous bromide solutions to bromate. Electrochim. Acta 29, 875-885 (1984). 